\title{
On computations of temperature dependent incompressible flows by high order methods
}

\author{
Jan $\operatorname{Pech}^{1, \text { a }}$ \\ ${ }^{1}$ Institute of Thermomechanics AS CR, v. v. i., Dolejškova 1402/5, Praha 8, 182 00, Czech Republic
}

\begin{abstract}
Parallel vortex shedding from the heated cylinder as dependent on the cylinder temperature is investigated in numerical simulation. The computational scheme for the system of the incompressible Navier-Stokes equations coupled with the heat equation is presented. The scheme is based on the mixed implicit-explicit solver of the third order and the spectral element method for the spatial approximation. The results are compared with the experiments for flow of air and water. The Strouhal-Prandtl-Reynolds relationship and evolution of the separation angle as dependence on the temperature is the final result of the computations.
\end{abstract}

\section{Introduction}

The vortex shedding from the circular cylinder is widely studied phenomena both experimentally and in the numerical simulations. This, geometrically simple, problem demonstrates various effects in the transition of the flow from the laminar to turbulent behaviour. From the wide literature concerning this problem we have already knowledge about the dependence of the vortex shedding frequency on the velocity magnitude and vortical structures in the wake. In the present study we restrict our attention to the flow regime, where the vortex shedding parallel to the cylinder occurs, and where a 2D numerical simulation is justified. This regime is achievable in the range of Reynolds numbers $R e_{c}<R e<160$, if $R e_{c}$ denotes the critical Reynolds number, separating the steady and unsteady flow. In this flow regime we study the influence of the cylinder wall heating to the vortex shedding frequency (described in terms of the Strouhal number $S t$ ). The Reynolds number is here defined as $R e=\frac{\left|\mathbf{u}_{\infty}\right| D \rho_{\infty}}{\mu_{\infty}}$, where $D$ stands for the cylinder diameter, $\mu$ is the dynamic viscosity, $\rho$ density, $|\mathbf{u}|$ the characteristic velocity magnitude and subscript $\infty$ denotes values in the far-field/inlet. The Strouhal number is defined as $S t=\frac{f D}{\left|\mathbf{u}_{\infty}\right|}$, where $f$ is the frequency of vortex shedding.

The fluids considered are water and air. For our study is most important that these two substances exhibit opposite temperature dependence of its viscosities. We expect the flow to be incompressible in case of both studied fluids, because the velocity magnitudes satisfy $M^{2} \ll 1(M$ is the Mach number). Effects of the buoyancy and viscous heating are neglected, since it has a minor influence to the flow in our setting. Therefore the influence of the flow by the temperature distribution is given by the temperature variation of the dynamic viscosity $\mu$ and the thermal conductiv-

\footnotetext{
a e-mail: jpech@it.cas.cz
}

ity $\lambda$. Frequency of the vortex shedding depends mostly on the viscosity, but variability in the thermal conductivity is also important, especially for preserving the correct local value of the Prandtl number $\left(\operatorname{Pr}=c_{p} \mu_{\infty} / \lambda_{\infty}\right)$, which moderates the heat equation. For the isothermal case, when the cylinder wall has the same temperature as the inlet fluid, the results of numerical simulation using the scheme of [1] give excellent agreement with $S t$-Re relationship (see e.g. [2]). For the study of the heating effects to the $S t-R e$ relation, the scheme for isothermal case is enhanced using technique already proposed in [3] and applied in the same manner to the heat equation as in [4]. In comparison to the previous results of [4] using the first order scheme, we present the third order scheme and optimise the computation in the spatial solver.

The flow parameters were chosen to correspond with those of [5], where an influence of heating to the Strouhal number was studied experimentally. The Strouhal number obtained from our computation in comparison to the values from the experiment then provides a basic information about the relevance of the computational results.

\section{Physical model}

A system of equations describing the motion and temperature distribution of an incompressible fluid with the temperature dependent viscosity and thermal conductivity may be written in the dimensionless form as

$$
\begin{gathered}
\frac{\partial \mathbf{u}}{\partial t}+(\mathbf{u} \cdot \nabla) \mathbf{u}=-\nabla p+\nabla \cdot\left[\frac{1}{\operatorname{Re}} \frac{\mu(T)}{\mu_{\infty}}\left(\nabla \mathbf{u}+(\nabla \mathbf{u})^{\mathrm{T}}\right)\right] \\
\nabla \cdot \mathbf{u}=0 \\
\frac{\partial T}{\partial t}+(\mathbf{u} \cdot \nabla) T=\nabla \cdot\left(\frac{1}{\operatorname{Pr} \operatorname{Re}} \frac{\lambda(T)}{\lambda_{\infty}} \nabla T\right)
\end{gathered}
$$

where $\mathbf{u} \in[0: 1]$ denotes velocity, $p$ is dimensionless kinematic pressure, $\mu(T)$ is the temperature dependent dy- 
Table 1. The power-law exponents of the viscosity and therm. conductivity of water and air used in the present computations.

\begin{tabular}{lll}
\hline & $M$ & $L$ \\
\hline water & -7.0 & 0.71 \\
air & 0.7774 & 0.85 \\
\hline
\end{tabular}

namic viscosity and $\lambda(T)$ is the thermal conductivity. Denoting by $T_{W}$ the temperature of the cylinder wall we have set $T \in\left[1: T_{W} / T_{\infty}\right]$.

To complete above mathematical system the functions $\lambda(T)$ and $\mu(T)$ are approximated as power laws

$$
\begin{aligned}
& \lambda(T)=\lambda_{\infty}\left(\frac{T}{T_{0}}\right)^{L} \\
& \mu(T)=\mu_{\infty}\left(\frac{T}{T_{0}}\right)^{M}
\end{aligned}
$$

based on the data [6] and procedure suggested in [2], respectively. Exponents $M$ and $L$, used in the computations, are listed in the table 1.

The whole system is fully coupled by the variable properties. The technique used in the numerical scheme decouples the system as the operator splitting method together with linearization and results in acceptably accurate and fast solver.

\section{Numerical methods}

The computational scheme for the system (1)-(3) originate from the high order splitting scheme [1] enhanced by the approach for variable viscosity ([3]) and was already presented in its first-order version in [4].

The temperature dependent properties of viscosity and thermal conductivity are divided into a constant and variable (subscript $s$ ) part

$$
\mu(T)=\mu_{\infty}+\mu_{s}(T), \lambda(T)=\lambda_{\infty}+\lambda_{s}(T) .
$$

The terms with variable parts $\left(\mu_{s}, \lambda_{s}\right)$ are left to the explicit evaluation together with the convective non-linearities. The constants $\mu_{\infty}$ and $\lambda_{\infty}$ stay on the place as in the constantproperty system.

Introducing a notation of a function value at the $n$-th time step, $f(n \Delta t)=[f]^{n}$, we can write the explicit part of the scheme for the incompressible Navier-Stokes equations

$$
\begin{aligned}
& \frac{\gamma_{0} \hat{\mathbf{u}}-\sum_{q=0}^{2} \alpha_{q}[\mathbf{u}]^{n-q}}{\Delta t}= \\
& =\sum_{q=0}^{2} \beta_{q}\left[\nabla \cdot\left(\frac{\mu_{s}(T)}{\operatorname{Re} \mu_{\infty}}\left(\nabla \mathbf{u}+(\nabla \mathbf{u})^{\mathrm{T}}\right)\right)-(\mathbf{u} \cdot \nabla) \mathbf{u}\right]^{n-q} .
\end{aligned}
$$

Evaluation of the viscosity follows $\left[\mu_{s}(T)\right]^{n}=\mu_{s}\left([T]^{n}\right)$ and coefficients $\alpha_{q}, \beta_{q}$ are listed in the table 2 .

The pressure Poisson equation as known from the fractional step methods follows

$$
\nabla^{2}[p]^{n+1}=\nabla \cdot\left(\frac{\hat{\mathbf{u}}}{\Delta t}\right)
$$

Table 2. The coefficients of the third-order IMEX scheme. For details we refer to [7], pg.264.

\begin{tabular}{llll}
\hline $\mathrm{q}$ & $\alpha_{q}$ & $\beta_{q}$ & $\gamma_{0}$ \\
\hline 0 & 3 & 3 & $11 / 6$ \\
1 & $-3 / 2$ & -3 & \\
2 & $1 / 3$ & 1 & \\
\hline
\end{tabular}

and is accompanied with the high order pressure boundary condition (HOPBC), which has crucial impact on the final accuracy of the scheme

$$
\begin{aligned}
& \frac{\partial[p]^{n+1}}{\partial \mathbf{n}}= \\
& =\mathbf{n} \cdot \sum_{q=0}^{2} \beta_{q}\left[\nabla \cdot\left(\mu(T)\left(\nabla \mathbf{v}+(\nabla \mathbf{v})^{\mathbf{T}}\right)\right)-(\mathbf{v} \cdot \nabla) \mathbf{v}\right]^{n-q}
\end{aligned}
$$

The condition in the form 9 expects a constant velocity on the boundary, what is satisfied in our model. The second step (8) allows use of the spectral element method as the spatial solver. It also applies the incompressibility to the second intermediate velocity field $\hat{\hat{\mathbf{u}}}$, which satisfy

$$
\frac{\hat{\mathbf{u}}-\hat{\mathbf{u}}}{\Delta t}=-\nabla p^{n+1}
$$

and finally forms the right hand side of the Helmholtz equation for the velocity at the new time step

$$
\left(\frac{1}{R e} \nabla^{2}-\frac{\gamma_{0}}{\Delta t}\right)[\mathbf{u}]^{n+1}=\frac{\gamma_{0}}{\Delta t} \hat{\hat{\mathbf{u}}}
$$

The temperature dependent thermal conductivity results in a strongly non-linear heat equation, which is linearized and splitted in the same manner as the equation of motion. The operator splitting is applied to perform linear terms implicitly and the non-linearities, containing convective term and variable part of the diffusion term, are evaluated explicitly. The explicit step of the third-order scheme then results in

$$
\begin{aligned}
& \frac{\gamma_{0} \hat{T}-\sum_{q=0}^{2} \alpha_{q}[T]^{n-q}}{\Delta t}= \\
& =\sum_{q=0}^{2} \beta_{q}\left[-(\mathbf{v} \cdot \nabla) T-\frac{1}{\operatorname{Pr} \operatorname{Re}} \nabla \cdot\left(\frac{\lambda_{s}(T)}{\lambda_{\infty}} \nabla T\right)\right]^{n-q} .
\end{aligned}
$$
tion

Implicit part has again the form of the Helmholtz equa-

$$
\left(\frac{1}{\operatorname{Pr} \operatorname{Re}} \nabla^{2}-\frac{\gamma_{0}}{\Delta t}\right)[T]^{n+1}=-\frac{\hat{T}}{\Delta t} .
$$

and is solved by the spectral element method.

\section{Results}

The computational domain with dimensions measured from the center of the cylinder had $51 D$ upstream, $100 D$ downstream and $51 D$ on both sides from the cylinder. A refinement towards the cylinder and the area of the wake was 


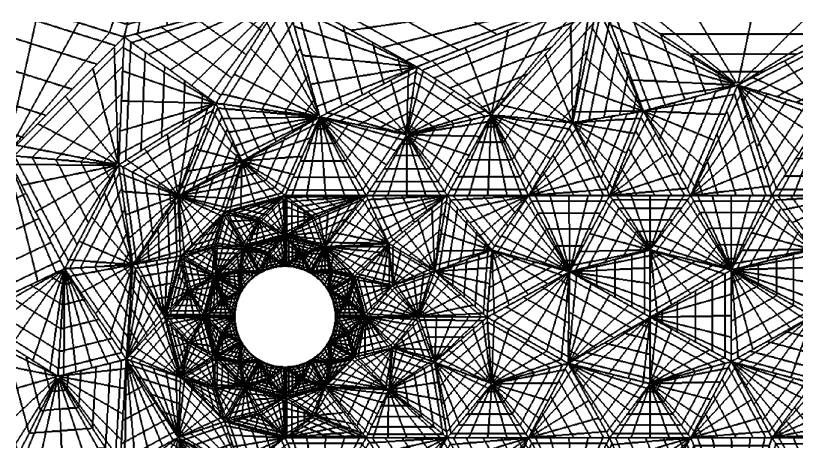

Fig. 1. Detail of the computational mesh around the cylinder. The quadrature points are connected by lines, so the particular triangular elements may be recognized. In the direction of converging lines the Gauss-Radau quadrature is used and Gauss-Lobatto rule is on the opposite side. The mesh, coarse in comparison to those of low order methods, has curved boundaries at the cylinder surface.

designed to keep a satisfactory spatial resolution. A detail of the cylinder area of the mesh consisting of 2360 triangular elements is in figure 1. Polynomial basis of order 6 was chosen as sufficient after convergence tests.

The computational scheme (7)-(13) was implemented on base of the Nektar++ library [8], a C++ library for implementation of the schemes based on the spectral element methods. Among the output quantities were positions of the zero shear stress on the cylinder boundary and values of the drag $C_{D}$ and lift $C_{L}$ coefficients

$$
C_{D}=\frac{2 F_{1}}{\rho\left|\mathbf{u}_{\infty}\right|^{2} D}, C_{L}=\frac{2 F_{2}}{\rho\left|\mathbf{u}_{\infty}\right|^{2} D} .
$$

Here we denoted

$$
\mathbf{F}=\left(\begin{array}{l}
F_{1} \\
F_{2}
\end{array}\right)=\int_{C}(\mathbb{T} \mathbf{n}) \mathrm{d} S,
$$

$C$ the cylinder surface and $\mathbb{T}=-p \mathbb{I}+\mu\left(\nabla \mathbf{u}+(\nabla \mathbf{u})^{\mathrm{T}}\right)$ the stress tensor. The shear stress was computed as a tangential component of the velocity gradient in the direction of the normal $\mathbf{n}$ to the cylinder wall

$$
\frac{\partial \mathbf{u}}{\partial \mathbf{n}} \cdot \mathbf{t}
$$

Values of $C_{D}, C_{L}$ and positions of all detected zeros of the shear stress were saved in every time step.

\subsection{Temperature dependence of St-Re relationship}

If $R e>R e_{c}$, a disturbances from initialisation of the computational process are not suppressed and the flow develops to the vortex shedding regime. History of $C_{D}$ and $C_{L}$ reflects the evolution of the flow and must be processed, since only the fully developed vortex street contains the frequency, which refers to the Strouhal number. The resulting $S t$-Re dependencies for both air and water for various temperatures of the cylinder are in the figure 2 and figure 3. The Strouhal number decreases with temperature

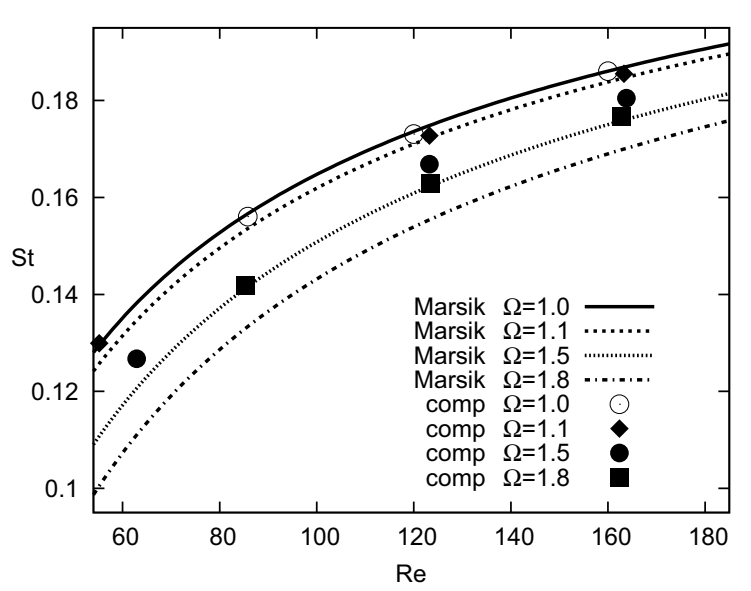

Fig. 2. The Strouhal-Reynolds number relationship for flow of air in isothermal case $\Omega=1.0$ and three heated cases $\left(\Omega=T_{W} / T_{\infty}\right)$. Results of computations are denoted by comp, curves Marsik are plots of eq.(4.4) from [2].

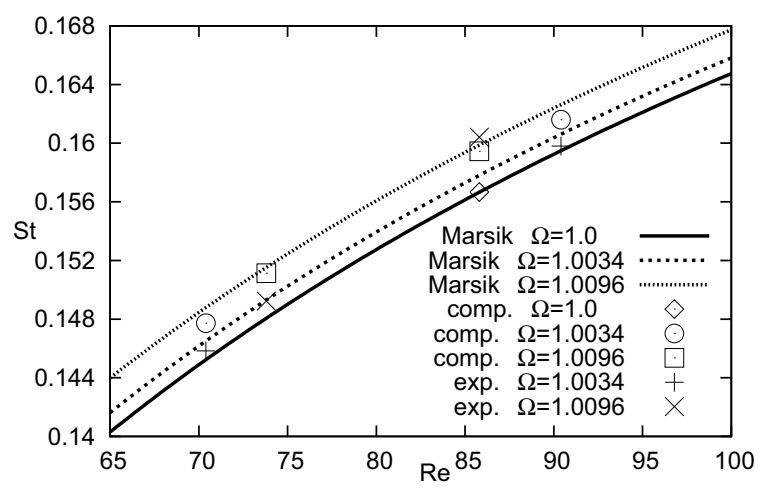

Fig. 3. Strouhal-Reynolds number relationship for flow of water. Experimental results of [5] are denoted as exp, other notation is adopted from figure 2 .

increment in case of air and increases in case of water. Presently computed data coincide with those computed in [4] by the polynomial approximation up to order 49. The new results therefore support applicability of very high order spatial approximations and shows that distinction of the computation from the experiment is not given by the spatial approximation with mentioned settings.

\subsection{Influence of heating to the separation angle}

The separation angle $\Theta$ is measured on the arc defined by frontal stagnation point, cylinder center and the separation point (figure 4). There are four positions of the zero shear stress on the cylinder, which correspond to frontal and backward stagnation points and points of separation on the "top" and "bottom" side of the cylinder. Obtaining the position of any of the zero points stayed on interpolation of the zero between two quadrature points with opposite sign of the shear stress value. The position of all four points oscillates with the frequency of the vortex shedding. Re- 


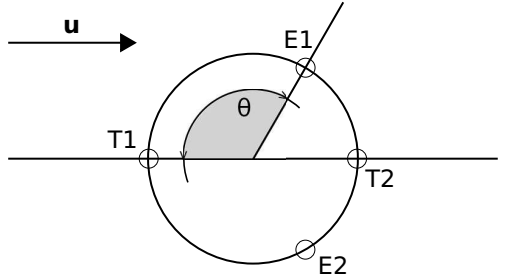

Fig. 4. The separation angle $\Theta$ is measured from the upstream zero shear stress point. Approximative positions on frontal (T1), backward (T2) stagnation points and two separation points (E1, E2) is drawn.

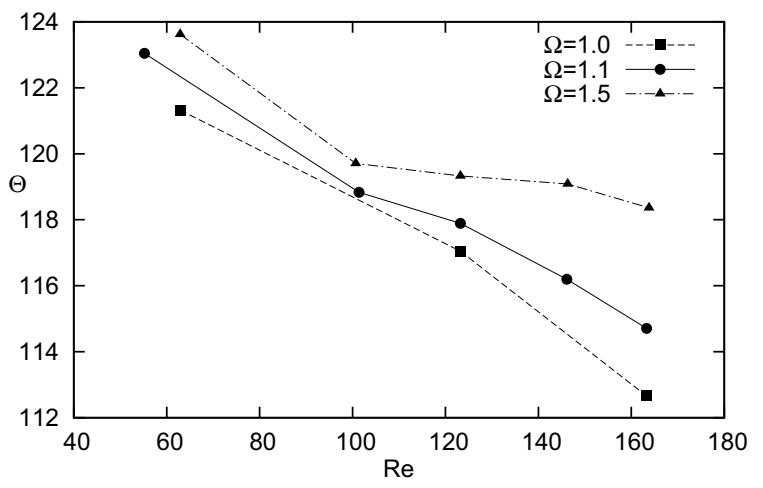

Fig. 5. $\Theta-R e$ dependence for various temperature ratios $\Omega=$ $T_{W} / T_{\infty}$. The values of separation angles are the mean values in time. For the maximal and minimal values observed we refer to figure 6 .

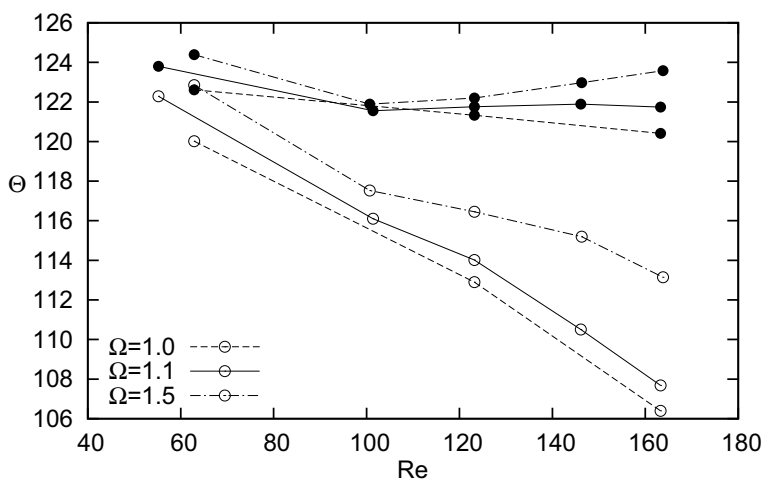

Fig. 6. Temperature dependence of amplitude of separation angle for various ratios of flow and cylinder temperatures $\Omega=T_{W} / T_{\infty}$. Filled points refers to maximal separation angles, empty points denotes the minima.

sulting angles as dependent on the Reynolds number and temperature are plotted in figure 5 and figure 6 .

The frontal stagnation point very accurately preserves mean value of $\Theta=0$ and oscillates with moderate amplitude $<2$ degrees. Time-averaged positions of the separation points (E1 and E2 in figure 4) appear symmetrically $\left(\Theta_{S 1} \simeq 360-\Theta_{S 2}\right)$. A difference between angles evaluated on the present mesh and mesh for very high order basis of [4] was observed. This deviation in results is up to 2 angular degrees and suggests a need of improvement of the present evaluation method, whose accuracy depends on lo- cal distribution of quadrature points, which is non-uniform over the elements.

\section{Conclusion}

There are still various settings in the computation, which need to be revised in relation to the results of the experiment. Values of the final Strouhal number are strongly influenced by the choice of the reference temperature and dependencies of $\mu$ and $\lambda$. Even though the buoyancy effects are negligible, the model expecting a constant density is limiting for the case of the heated flows even if the velocity magnitudes in the flow are small. Successes of the present computations are in confirmation of relevance of the previous very high order computations [4] and inclusion of the variable properties into the solver capable of the fast and accurate computations of the fluid flow. Results of the computations clearly shows the impacts of heating in both settings with properties of water and air. Accuracy of the computation is comparable with tolerance of the experimental data. Observed deviation of the absolute values of computation and experiment are left for wider discussion. Computations of the separation angle shows its dependence on temperature and confirms its dependence on the flow velocity. Observation of this quantity has many complications in experimental studies and the computation clarifies the phenomenon especially in its time dependence.

\section{Acknowledgement}

Institutional support RVO: 61388998 is gratefully acknowledged.

\section{References}

1. G.E. Karniadakis, M. Israeli, S. A. Orszag, J. Comput. Phys. 97, 414-443 (1991)

2. F. Maršík, Z. Trávníček, R.-H. Yen, W.-Y. Tu, A.-B. Wang, Proceedings of CHT-08 2008

3. G. Karamanos, S. Sherwin, Appl. Numer. Math. 33, 455-462 (2000)

4. J. Pech, Programs and Algorithms of Numer. Math. 17, 169-174

5. T. Vít, M. Ren, Z. Trávníček, F. Maršík, C. Rindt, Exp. Therm. Fluid Sci. 31, 751-760

6. B. Gebhart, Heat conduction and mass diffusion (McGraw-Hill, Inc., 1993)

7. G.E. Karniadakis, S. Sherwin, Spectral/hp Element Methods for CFD (Oxford University Press, New York, 2005)

8. C.D. Cantwell, et al., Comput. Phys. Commun. 192, 205-219 (2015) 